\title{
Studies of Carbohydrate and Lipid Metabolism in Women Developing Hypertension on Oral Contraceptives
}

\author{
BRIDGETT MASON, NIGEL OAKLEY, VICTOR WYNN
}

British Medical fournal, 1973, 3, 317-320

\section{Summary}

Metabolic studies in 100 women developing hypertension on combined oestrogen-progestogen oral contraceptives have been compared with similar studies in normotensive women on oral contraceptives, matched for age and duration of contraceptive use, and in women not taking contraceptives.

The metabolic changes known to be induced by oral contraceptives-impaired glucose tolerance, elevated blood pyruvate levels, and raised serum lipid concentrations-were found to be exaggerated in the matched hypertensive group, largely due to pronounced abnormalities in 33 subjects with diastolic blood pressures over $110 \mathrm{~mm} \mathrm{Hg}$.

Women developing severe hypertension were older, more obese, and of higher parity than those with mild hypertension and there was a high incidence of previous toxaemia of pregnancy in the hypertensive group.

The results show that in women on oral contraceptives changes in blood pressure and in metabolic functions tend to be correlated with one another, and are consistent with the hypothesis that oral contraception induces a primary biochemical effect whose expression in the individual is determined by intrinsic factors including genetic constitution, age, weight, and parity.

\section{Introduction}

Combined oestrogen-progestogen oral contraceptives (O.C.) may cause a rise in blood pressure, raised levels of serum lipids, and impairment of carbohydrate tolerance. No association between the metabolic and vascular side effects has, however, yet been described. Small blood pressure rises occur in a high proportion of women given O.C. (Woods, 1967; Tyson, 1968; Kunin et al., 1969; Weir et al., 1971), while the reported incidence of hypertension (greater than 140/90 mm $\mathrm{Hg}$ ) arising in previously normotensive women given the preparation ranges from less than 2\% (Weir et al., 1971) to $18 \%$ (Saruta et al., 1970). The mechanism of the blood pressure rise is uncertain but raised renin substrate levels may contribute (Newton et al., 1968; Crane and Harris, 1969; Skinner et al., 1969; Laragh, 1970), though Catt et al. (1971) were unable to correlate plasma angiotensin II levels with blood pressure changes in women on O.C. Walters and Lim (1969) suggested that increased cardiac output might be an important factor which would mainly raise the systolic blood pressure. Spellacy and Birk 1972) found that O.C. containing synthetic oestrogens, and these compounds given alone, induced blood pressures of over $140 / 90 \mathrm{~mm} \mathrm{Hg}$ in $5-7 \%$ of women when given for six months. Conjugated oestrogens (Premarin) induced diastolic hypertension comparable to that seen in women on O.C., but

\footnotetext{
Alexander Simpson Laboratory for Metabolic Research, St. Mary's Hospital Medical School, London W.2

BRIDGETT MASON, M.B., Clinical Assistant

NIGEL OAKLEY, M.B., M.R.C.P., Senior Lecturer in Human Metabolism

VICTOR WYNN, M.D., F.R.C.PATH., Professor of Human Metabolism, Director
}

without systolic changes. Progestogens given alone lowered diastolic blood pressure. These observations suggest that it is the oestrogen component of the pill which causes hypertension.

Wynn et al. (1969) showed that mean fasting triglyceride and cholesterol levels are both significantly raised in women on O.C., the increase in triglyceride being related to the oestrogen content of the pill and that of the serum cholesterol being most distinct in women taking preparations with the highest progestational activity (Stokes and Wynn, 1971).

Reports of changes in carbohydrate tolerance on O.C. have given a widely varying incidence of abnormalities. Possible reasons, including patient selection, have recently been reviewed (Adams and Oakley, 1972). Wynn and Doar (1969) found that glucose tolerance deteriorated in $78 \%$ of women given O.C., $13 \%$ developing chemical diabetes after three months. Spellacy et al. (1970) found that after eight years on O.C. $39 \%$ of women had relatively impaired and $39 \%$ frankly abnormal glucose tolerance. Di Paola et al. (1968) found abnormal results in $67 \%$ and Javier et al. (1968) in $88 \%$ of women on O.C. who were given glucocorticoids before the test. Similarities in glucose and pyruvate metabolism between women on O.C., obese subjects, and those with glucocorticoid excess (Doar and Wynn, 1970) suggest that the latter may be a factor common to all these situations. Glucocorticoid excess may also be associated with hypertension, while an association between diabetes and hypertension independent of a thiazide effect has been reported (Drazin, 1953, Kobyashi, 1959). The presence or absence of O.C.-induced abnormalities in individual women must reflect susceptibility to, for instance, hypertension or diabetes. Nevertheless, if different pill complications reflect a single underlying biochemical lesion, some association between these abnormalities may be expected, reflecting the severity of the biochemical changes. A study was therefore designed to discover whether there is any association between hypertension and metabolic side effects in women taking O.C. An attempt has also been made to identify the factors predisposing women on combined O.C. to increased blood pressure.

\section{Subjects}

From women already being followed up in our own department, and in response to a request to family planning clinics for referral of women developing hypertension on O.C., a group of 100 women was collected in whom resting blood pressures of $140 \mathrm{~mm} \mathrm{Hg}$ systolic and $90 \mathrm{~mm} \mathrm{Hg}$ diastolic or more were obtained on at least two occasions (group H.T.; hypertension). This group was subdivided into 67 women with diastolic blood pressures from $90-110 \mathrm{~mm} \mathrm{Hg}$ (group M.H.T.; mild hypertension) and 33 in whom a diastolic pressure of over $110 \mathrm{~mm} \mathrm{Hg}$ was recorded on at least one occasion (group V.H.T.; severe hypertension). Thirty-five women stopped O.C. because of hypertension and were studied a second time at least three months after changing their method of contraception (groups On and Off).

For comparison, two groups of women were selected. The first consisted of 91 women tested under identical conditions before starting oral contraception (group' Pre); details of this group have already been published (Wynn and Doar, 1969). The second group consisted of 100 women who had been similarly investigated and whose blood pressures had remained within normal limits on O.C. (group N.T.; normotensive). This latter control group of women on O.C. was selected using a com- 
puterized information retrieval system which has been developed for O.C. studies in our unit; the normotensive women in this group were matched with the hypertensive group for age and duration of O.C. use.

\section{Methods}

Blood pressure was measured with a conventional mercury sphygmomanometer after the subject had been sitting at rest for at least 10 minutes. The pressure at which muffling occurred on auscultation was taken as the diastolic end point. All women were asked to take at least $200 \mathrm{~g}$ carbohydrate a day (according to a printed sheet) for at least three days before their glucose tolerance test. This was carried out fasting, using oral glucose $1 \mathrm{~g} / \mathrm{kg}$, venous blood samples being taken half-hourly for three hours from an indwelling needle. Duplicate fasting samples were taken about 15 minutes apart, before giving glucose. Plasma glucose was estimated by an automated glucose oxidase method (Cramp, 1967). Glucose tolerance was assessed using the area under the three-hour curve (Wynn and Doar, 1966) recently shown to be the most reproducible parameter of oral glucose tolerance in the assessment of borderline diabetes (Harding et al., 1973). Blood pyruvate levels were estimated half-hourly up to two hours using the method of Cramp (1968). Serum cholesterol and triglyceride were estimated on fasting samples using automated fluorometric techniques (Cramp and Robertson, 1968; Robertson and Cramp, 1970). The statistical significance of differences between patient groups has been calculated using Student's $t$ test and the $x^{2}$ test as appropriate.

\section{Results}

Comparison of Patient Groups.-Groups H.T. and N.T. were matched for age and duration of O.C. use. Despite the fact that no other matching procedures were used, no differences between these groups could be detected for any of the parameters examined (table I). It was our impression, however, that in comparison to the average woman on O.C. women in group H.T. tended to be older, more parous, and more overweight. This was further studied by examining the patient characteristics of the two subgroups (M.H.T. and V.H.T.). Compared to group M.H.T. patients in group V.H.T. were older $(P<0.005)$, more obese $(P<0.005)$, and of higher parity $(P<0.1)$. There was no significant difference between M.H.T. and V.H.T. patients in duration of O.C. use, family history of diabetes or hypertension, or incidence of previous toxaemia of pregnancy. Unfortunately, reliable information on previous toxaemia was not available for the N.T. group, but the $21 \%$ and $30 \%$ incidence in groups M.H.T. and V.H.T. respectively is substantially higher than the incidence of about $5 \%$ reported for all pregnancies (Dewhurst, 1972), though of the same order as that reported in another small series of women developing hypertension on O.C., and for a small group of women with essential hypertension (Weinberger et al., 1969).

Fasting Plasma Glucose.-Mean fasting plasma glucose values for groups M.H.T. and V.H.T. were both significantly higher than for group N.T. $(P<0.001)$ though the two hypertensive groups did not differ significantly from one another (table II). The group of women tested before starting O.C. (Pre) yielded a mean value similar to group N.T. whose blood pressures had not altered on O.C. Fasting plasma glucose fell, but not significantly, in the group retested after stopping O.C. (On and Off).

Glucose Tolerance.-The lowest mean glucose area, seen in group Pre (fig. 1), was significantly below that seen in group N.T. $(P<0.001)$. The mean value for group M.H.T. was almost identical to that of the N.T. group, but severer hypertension (V.H.T.) was associated with significantly greater impairment of glucose tolerance $(P<0.001)$. There was a significant fall in glucose area between groups On and Off $(\mathbf{P}<$ 0.001 ) showing that the abnormality is usually reversible.

Fasting Blood Pyruvate Levels.-There was a clear difference $(P<0.001)$ between groups Pre and N.T., and the total hyper-

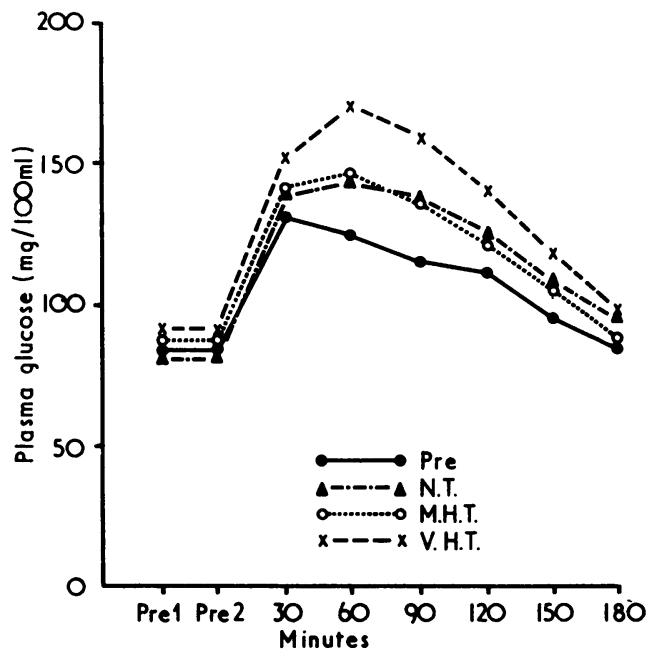

FIG. 1-Mean oral glucose tolerance profiles. (For definitions of group abbreviations see text.)

TABLE I-Clinical Characteristics of Subjects Studied. (For Definitions of Group Abbreviations see Text)

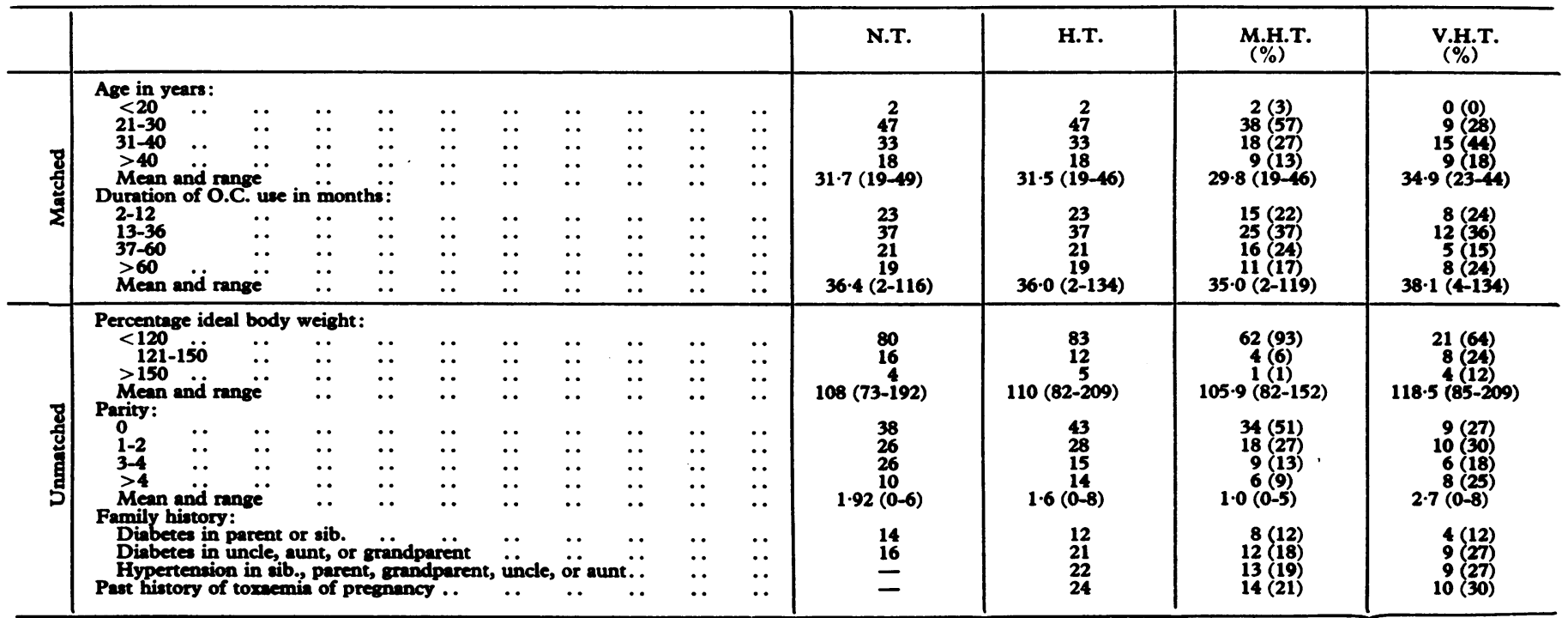


TABLE II-Mean Glucose, Pyruvate, and Lipid Values in Various Groups

\begin{tabular}{|c|c|c|c|c|c|c|c|c|c|}
\hline \multirow{3}{*}{\multicolumn{3}{|c|}{ Subject Group }} & \multirow{3}{*}{ No. of Women } & \multirow{3}{*}{$\begin{array}{l}\text { No. of Women } \\
\text { with O.G.T.T. } \\
\text { Area }>800 \text { units }\end{array}$} & \multicolumn{5}{|c|}{ Mean Values \pm 1 S.E. of Mean } \\
\hline & & & & & \multirow{2}{*}{$\begin{array}{c}\text { O.G.T.T. Area } \\
\text { (in arbitrary units) }\end{array}$} & \multicolumn{4}{|c|}{ Fasting $(\mathrm{mg} / 100 \mathrm{ml})$} \\
\hline & & & & & & Glucose & Pyruvate & Triglyceride & Cholesterol \\
\hline Pre & . & .. & 91 & $7(8 \%)$ & $663 \pm 9 \cdot 4$ & $82.8 \pm 0.92$ & $0.59 \pm 0.02$ & $67 \cdot 2 \pm 2 \cdot 29$ & $179 \pm 3 \cdot 20$ \\
\hline N.T. & .. & .. & 100 & $32(32 \%)$ & $741 \pm 11 \cdot 7$ & $81.6 \pm 0.78$ & $0.70 \pm 0.01$ & $108.7 \pm 3.55$ & $194.6 \pm 3.78$ \\
\hline & & $\begin{array}{l}\cdots \\
\cdots \\
\ldots\end{array}$ & & $\begin{array}{l}23(33 \%) \\
43 \%) \\
20(60 \%)\end{array}$ & $\begin{array}{l}.742 \pm 15.6 \\
\pm 14.5 \\
842 \pm 21.5 \\
\end{array}$ & $\begin{array}{l}.86 .8 \pm 1.18 \\
\pm 1.04 \\
87.6 \pm 2.08 \\
\end{array}$ & $\begin{array}{l}0.76 \pm 0.02 \\
0.02 \\
0.80 \pm 0.05\end{array}$ & $\begin{array}{l}110.7 \pm 11.20 \\
\pm 8.08 \\
121.7 \pm 8.72\end{array}$ & $\begin{array}{l}193 \cdot 8 \pm 5 \cdot 28 \\
\pm 4 \cdot 26 \\
202 \cdot 5 \pm 7 \cdot 17\end{array}$ \\
\hline On ... & .. & .. & 35 & $23(66 \%)$ & $836 \pm 24 \cdot 0$ & $87.6 \pm 1.58$ & $0.79 \pm 0.05$ & $124.9 \pm 8.00$ & $210 \pm 7 \cdot 76$ \\
\hline Off & .. & .. & 35 & $7(20 \%)$ & $728 \pm 17 \cdot 9$ & $84 \cdot 6 \pm 1 \cdot 25$ & $0.72 \pm 0.04$ & $86.8 \pm 4.63$ & $203 \pm 6 \cdot 71$ \\
\hline
\end{tabular}

O.G.T.T. = Oral glucose tolerance test.

tensive group (H.T.) differed significantly $(P<0.05)$ from group N.T. (table II). However, while subdivision of hypertensive women into groups M.H.T. and V.H.T. showed that the severer hypertension tended to be associated with a higher fasting pyruvate level, the smaller size of the hypertensive subgroups led to loss of significance in their difference from the normotensive controls.

Pyruvate Changes after Glucose.-Blood pyruvate levels after glucose closely reflected the differences seen in the fasting state. The shape of the curves was similar for all the groups studied (fig. 2), and increasing blood pressure was associated with a progressive rise in mean plasma pyruvate above that already seen in group N.T. Differences between the H.T. and N.T. groups reached a significance level of $P<0.1$ throughout the test, and this was not improved by separate consideration of subgroups M.H.T. and V.H.T.

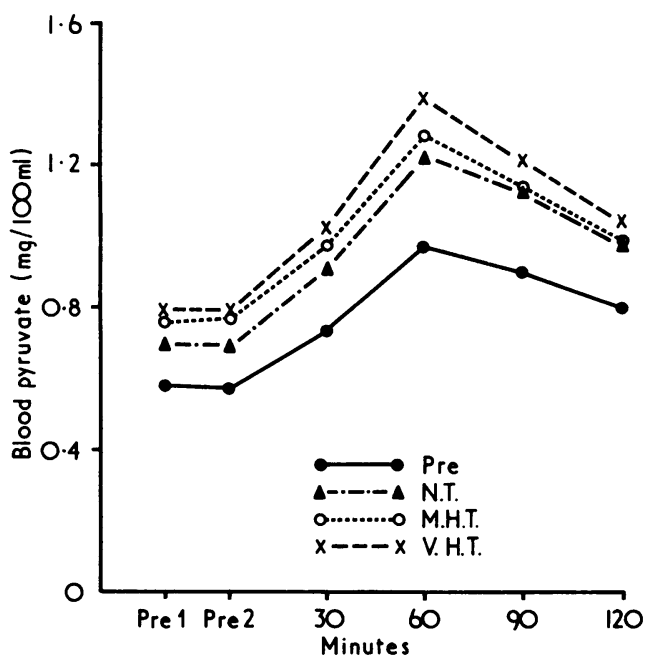

FIG. 2-Mean blood pyruvate values after oral glucose.

Serum Triglyceride and Cholesterol.-An expected increase in both serum triglyceride and cholesterol $(P<0.001)$ was seen between groups Pre and N.T. (table II). Values for group M.H.T. agreed closely with those for N.T. but mean values for the V.H.T. group were higher, though for neither estimation did the difference between V.H.T. and M.H.T. quite reach statistical significance.

\section{Discussion}

Previous studies from our own and other laboratories have shown that oestrogen-progestogen oral contraceptives cause impairment of glucose tolerance, raised fasting and postglucose blood pyruvate levels, and a rise in fasting serum cholesterol and triglyceride (Hazzard et al., 1969; Wynn and Doar, 1969; Spellacy et al., 1970; Stokes and Wynn, 1971). These changes are aggravated by a coexistent obesity. The present study suggests that the severer degrees of hypertension in women on combined oral contraceptives are also associated with an exaggeration of the metabolic changes normally seen in women on the pill.

In this study the hypertensive subjects were initially compared with a normotensive group of women on oral contraceptives, against whom they were matched with respect to age and duration of use of the preparation. This was done in order to detect correlations between hypertension and metabolic abnormalities due to factors other than those for which the groups were matched. Differences between the total hypertensive group and the matched group of normotensive pill users were found for fasting glucose and pyruvate, and for glucose and pyruvate levels after glucose. Fasting serum lipids showed a moderate rise in the hypertensive as compared to the normotensive group but differences were not statistically significant.

The average age of women in the hypertensive group, and therefore those who were normotensive, was higher than might have been expected in an unselected group of women on combined oral contraceptives, and it therefore seemed possible that at least one of the selected matching functions actually predisposed to hypertension. Subdivision of the hypertensive group into women with mild hypertension and severe hypertension provided evidence on this point. Failure to find differences between the mild and the severe hypertension groups for age or duration of use of the pill would have indicated that these factors were not positively related to the severity of hypertension induced by oral contraceptives. In fact mean age, degree of obesity, and parity were greater for the severe than for the mild hypertension group, but duration of pill use was not. The findings suggest that age, degree of obesity, and parity are all correlated with development of severe hypertension, but that duration of pill use is not. It is, however, difficult to establish whether it is age, weight, or parity that is of primary importance as these factors are related variables. It is also important to note that all three are factors which tend to predispose to abnormal carbohydrate tolerance (Pyke and Please, 1957). These findings explain the relatively high average age of the matched groups-just over 31 years as compared with 26 years for a pre-pill group and 32 years for a group of women who had stopped the pill for various reasons (Wynn and Doar, 1969). In support of the present findings Clezy et al. (1972) have reported development of a significant correlation between systolic blood pressure with age in women on the pill. The mean degree of obesity found in the normotensives $(108 \%)$ and hypertensives $(110 \%)$ was greater than that of the pre-pill group $(102 \%)$, a difference to which age, parity, and pill use are likely to have contributed.

Examination of metabolic functions for the subgroups with mild and severe hypertension showed that while significant differences between the normotensive and severe hypertension 
groups were almost always found, there were few differences between women with mild hypertension and the normotensive controls. This could have been because only severer degrees of hypertension modify the metabolic abnormalities already present in patients on the pill. Alternatively, as the normotensive group was matched with the whole hypertensive population rather than the subgroups with mild or severe hypertension, the mild subgroup was on average younger and therefore less overweight and less parous than the normotensive group. This could have influenced metabolic functions independently and thus masked a small adverse metabolic effect truly associated with mild hypertension.

The most striking feature of the study is the uniform pattern of progressive abnormalities seen as groups of patients are followed from Wynn and Doar's (1969) pre-pill group at one extreme to the severe hypertensives at the other. Without exception, the same pattern is seen for fasting serum lipids and for blood glucose and pyruvate both before and after glucose loading. This observation shows that the metabolic abnormalities seen in women who are hypertensive on combined oral contraceptives are qualitatively the same as those seen in women who are normotensive on the pill, and are therefore likely to be due to an exaggerated expression of these changes.

As in normotensive women, discontinuance of the preparation leads to progressive return of metabolic changes towards normal, though neither fasting plasma glucose, pyruvate, nor lipids, nor glucose area, had returned to normal at three months after stopping the pill. It is consistent with clinical experience that these metabolic functions, and also blood pressure, may take many months to come to pre-pill levels (Macintosh, 1968; Weinberger et al., 1969). This similarity between the time course of reversal of metabolic and blood pressure changes provides some further support for a common causation of the two effects.

The findings suggest that a number of different side effects of combined oral contraceptives may have a single biochemical basis, such as increased glucocorticoid activity. This leads to changes in blood pressure, weight, and metabolic functions which are statistically significant in large populations and clinically significant in particular individuals. The presence of clinically important abnormalities is determined both by the severity of the basic biochemical abnormality and by the presence of intrinsic predisposing factors. For metabolic abnormalities these may include genetic constitution, weight, age, and parity; while age, genetic factors, and previous toxaemia of pregnancy (Clezy et al., 1972) may be some of the factors acting in the case of blood pressure.
From the practical point of view, as both the hypertensive and metabolic effects of the pill may be atherogenic, older women of high weight and parity and those with a history of toxaemia of pregnancy should be supervised with particular care when given combined oral contraceptives.

We acknowledge the receipt of a grant from the Medical Research Council.

\section{References}

Adams, P. W., and Oakley, N. W. (1972). Clinics in Endocrinology and Metabolism, 1, 697.

Catt, K. J., et al. (1971). Lancet, 1, 459.

Clezy, T. M., Foy, B. N., Hodge, R. L., and Lumbers, E. R. (1972). British Heart Fournal, 34, 1238.

Cramp, D. G. (1967). Fournal of Clinical Pathology, 20, 910.

Cramp, D. G. (1968). Fournal of Clinical Pathology, 21, 171.

Cramp, D. G., and Robertson, G. (1968). Analytical Biochemistry, 25, 246.

Crane, M. G., and Harris, J. J. (1969. Fournal of Clinical Endocrinology and Metabolism, 29, 558 .

Dewhurst, C. J. (1972). Integrated Obstetrics and Gynaecology for Postgraduates, ed. C. J. Dewhurst, p. 269. Oxford, Blackwell Scientific.

Di Paola, G., Puchulu, F., Robin, M., Nicholson, R., and Marm, M. (1968). American fournal of Obstetrics and Gynecology, 101, 206.

Doar, J. W. H., and Wynn, V. (1970). British Medical fournal, 1, 149.

Drazin, M. L. (1953). Diabetes, 2, 433.

Harding, P, Oakley, N., and Wynn, V. (1973). Submitted for publication.

Hazzard, W. R., Spiger, M. J., Bagdade, J. D., and Bierman, E. L. (1969) New England fournal of Medicine, 280,471 .

Javier, Z., Gershberg, H., and Hulse, M. (1968). Metabolism, 17, 443.

Kunin, C. W. McCormack, R. C., and Abernathy, J. R. (1969). Archives of Internal Medicine, 123, 362.

Laragh, J. H. (1970). Circulation, 62, 983.

Macintosh, A. M. (1968). Medical fournal of Australia, 2, 1010.

Newton, M. A., Sealey, J. E., Ledingham, J. G. G., and Laragh, J. H. (1968). American fournal of Obstetrics and Gynecology, 101, 1037.

Pyke, D. A., and Please, N. W. (1957). Fournal of Endocrinology, 15, xxvi.

Robertson, G., and Cramp, D. G. (1970). Fournal of Clinical Pathology, 23, 243.

Saruta, T., Saade, G. A., and Kaplan, N. M. (1970). Archives of Internal Medicine, 126, 621 .

Skinner, S. L., Lumbers, E. R., and Symond, E. M. (1969). Clinical Science, 36, 67.

Spellacy, W. N., and Birk, S. A. (1972). American fournal of Obstetrics and Gynecology, 112, 912 .

Spellacy, W. N., Buhi, W. C., Spellacy, C. E., Moses, L. E., and Goldzieher, J. W. (1970). American fournal of Obstetrics and Gynecology, 106, 173. Stokes, T., and Wynn, V. (1971). Lancet, 2, 677.

Tyson, J. E. A. (1968). American fournal of Obstetrics and Gynecology, 100, 875.

Walters, W. A. W., and Lim, Y. L. (1969). Lancet, 2, 879.

Weinberger, M. H., Collins, R. D., Dowdy, A. J., Nokes, G. W., and Luetscher, J. A. (1969). Annals of Internal Medicine, 71, 891.

Weir, R. J., et al. (1971). Lancet, 1, 467.

Woods, J. W. (1967). Lancet, $2,653$.

Wynn, V., and Doar, J. W. H. (1969). Lancet, 2, 761 Wynn, V., and Doar, J. W. H. (1969). Lancet, $2,761$.
Wynn, V., Mills, G. L., Doar, J. W. H., and Stokes, T. (1969). Lancet, 2,

\title{
Prevalence of Tetracyclines in Children's Teeth- Study II: A Resurvey after Five Years
}

\author{
D. J. STEWART
}

British Medical fournal, 1973, 3, 320-322

\section{Summary}

The capacity of the tetracyclines to discolour teeth has been widely recognized for bver 10 years. This survey examined recent trends in tetracycline usage as shown Department of Child and Preventive Dentistry, The Queen's Uni-
versity, Belfast BT12 6BP

D. J. STEWART, M.D.S., F.F.D., Reader and Consultant by deposits of the antibiotic in primary molars extracted from 505 children aged from 3 to 5 years.

It was found that $\mathbf{7 0} \%$ of the children had been given the antibiotic during their first three years of life, each having received on average 2.4 courses. This represented an increase of $12 \%$ in tetracycline usage in children of this age as compared with a similar series five years ago. There was no evidence of increasing preference by prescribers for oxytetracycline (the analogue least likely to cause dental discoloration). Staining of the permanent incisor teeth frequently occurs if tetracyclines are given to children under 6 years of age and it is predicted that 